\title{
Enlightenment by the invisible
}

\author{
Peter Nick $^{1}$
}

Received: 19 August 2015 / Accepted: 19 August 2015

(C) Springer-Verlag Wien 2015

Light is the primary energy source for life on this planet and therefore also has turned into a primary signal for many organisms. As specific and advanced facet of this signalling aspect, light as physical base for microscopy has always been the primary source of information for cell biologists. In his Theory of Colours, Johann Wolfgang v. Goethe discussed the curious fact that light can only been perceived through its interaction with matter (he used the term Trübung, meaning turbidity). This is, by the way, true for other physical factors as well, may it be gravity, or may it be electric or magnetic fields. Always, the (physical) primary input has to be transformed into a (biochemical) signal that then can be sensed by the living organism. This transformation event has therefore been delineated as susception from the sensory event itself (Björkman 1988). As interesting consequence of the requirement for susception, physical factors can penetrate into organisms without leaving any trace except in those cells, where the susceptor is present and thus will generate a biochemical event. Two contributions to the current issue illustrate this principle for the case of light:

Roots, as organs dwelling in the dark, are certainly not expected to be primary recipients of light signals. However, a closer look reveals that the architecture of roots has to be adjusted with growth and development of the organs above ground. Especially during early development, this adjustment can be crucial for survival of the young seedling. As shown by Chen et al. (2015) in the current issue, the seminal roots of rice

Handling Editor: Peter Nick

\section{Peter Nick}

peter.nick@kit.edu

1 Molecular Cell Biology, Botanical Institute, Karlsruhe Institute of Technology, Karlsruhe, Germany seedlings develop strong nutations (manifest as wavy growth), when the seedling is irradiated by light. The biological function of this phenomenon seems to be linked with improved anchoring of the seedling by the spiralising seminal root, once the upper organs (especially the so called coleoptile) have reached the light. In a previous publication (Chen and Kao 2012), a role of the gas nitric oxide (NO) as regulator of root development could be demonstrated, leading to the hypothesis that NO might also act as second messenger transducing the effect of light on root nutations. Using a carefully designed set of treatments with NO donors or scavengers, along with a fluorescent dye recording the abundance of $\mathrm{NO}$ in the root of dark- or light-grown seedlings, they first show that NO is necessary and sufficient to evoke the nutations. In the next step, they test a potential interaction of this volatile messenger with either different phytohormones, or their respective inhibitors. From these combinatorial treatments, they can deduce the hierarchy of events triggered by the perception of light in the root tip (probably through the phytochrome photoreceptors), leading to the release of NO, which then will activate the synthesis of jasmonates that, in turn, will modulate the formation of ethylene (another volatile signal), and auxin transport, culminating in the enhanced nutational growth underlying the morphological response of the root. Although not been addressed in this work, light has long been discussed to travel in plant tissues by reflection within the vascular bundles, very similar to the way, how glass fibers efficiently transmit even spurious traces of light (Mandoli and Briggs 1982). Only when this invisible light hits the responsible photoreceptor in the root tip, the resulting conformational change will then release the chain of event leading to the morphogenetic response of the root.

The same principle - an invisible signal travels freely through the organism and is transformed into a visible signal at a specific region of interest — can also be exploited as 
technical tool for cell biology as shown in the contribution by Mizuta et al. (2015) in the current issue. They use nearinfrared light $(\lambda \sim 1000 \mathrm{~nm})$ for non-invasive imaging of intact Arabidopsis plants by two-photon excitation microscopy. The principle of this method is to use two lasers that oscillate in phase and are focussed on the region of interest deep in the observed tissue. The energy of the two photons will then be added up in the focal spot, providing the energy to excite a fluorescent probe, which can then be recorded by confocal microscopy. Because even the neighbouring cell will not perceive any input (since here the near-infrared photons will not add up, such that there is no biologically effective form of energy), the excitation is strictly confined to the cell that is actually imaged. This principle reduces the radiation load in the observed organism to the utmost minimum. While twophoton microscopy is already widely used in animal cell biology, for instance to follow the development of fish embryos in time and space, or to follow the activity of individual neurons in mouse brains in response to specific stimuli, the applications in the plant fields have remained limited. This is astonishing at first sight, because two-photon excitation microscopy has been successfully employed also in plant cells (Feijó and Moreno 2004). The reason for this limitation has to be sought in the strong autofluorescence of plant tissues. Autofluorescence is not only caused by activation of chlorophyll, which will provide a quite disturbing red background, but also by numerous secondary plant compounds, such as the phenylpropanoids. The authors circumvent this problem by using different fluorescent reporters such as TagRFP or tdTomato, whose activity is shifted to the red part of the visible spectrum. They succeed to substantially diminish nonspecific autofluorescence, and collect impressive, threedimensional images from the deep interior of intact, living, leaves, roots and sexual organs. This technical advance will extend the applicability of two-photon imaging in plant cell biology and thus allow to get a bit closer to the ideal (but principally impossible) situation of experimental systems that can be observed under "natural" conditions.

Conflict of interest The author declares that there is no conflict of interest.

\section{References}

Björkman T (1988) Perception of gravity by plants. Adv Bot Res 15:1-4 Chen YH, Kao CH (2012) Calcium is involved in nitric oxide- and auxininduced lateral root formation in rice. Protoplasma 249:187-195

Chen HW, Shao KH, Wang SJ (2015) Light-modulated seminal wavy roots in rice mediated by nitric oxide-dependent signaling. Protoplasma

Feijó JA, Moreno N (2004) Imaging plant cells by two-photon excitation. Protoplasma 223:1-32

Mandoli DF, Briggs WR (1982) Optical properties of etiolated plant tissues. Proc Natl Acad Sci USA 79:2902-2906

Mizuta Y, Kurihara D, Higashiyama T (2015) Two-photon imaging with longer wavelength excitation in intact arabidopsis tissues. Protoplasma 